\title{
Parvovirus B19 Presenting with Severe Sepsis in a Previously Healthy 25-year-old Female
}

\author{
Justin M. Bailey, MD
}

Family medicine physicians will encounter severe sepsis. Normally the work-up consists of searching for the bacterial source and then modifying initial empiric treatment. Unfortunately, not all infectious sources of sepsis are bacterial; 7\% of sepsis cases seen are due to a virus, fungi, or protozoa. ${ }^{1}$ Presented here is a case of an otherwise healthy adult female with severe sepsis, negative blood cultures, and a normal complete blood cell (CBC) count. Extensive work-up was highly suggestive of parvovirus B19 infection as the cause. MEDLINE review revealed no previously described cases.

\section{Case Presentation}

A 25-year-old previously healthy female presented to the emergency department with a chief complaint of dizziness and four days of fever to $105.5^{\circ} \mathrm{F}$. She was hypotensive to $80 / 40 \mathrm{~mm} \mathrm{Hg}$ and tachycardic to 120 beats per minute (bpm). After a $3 \mathrm{~L}$ fluid bolus and antipyretics were given, basic laboratory work and blood cultures were drawn, symptoms improved and the patient was discharged.

She returned to the emergency department $24 \mathrm{~h}$ later with recurrent fever and new symptoms of nausea and vomiting, edema in the upper and lower extremities, and a confluent macular erythematous rash over arms and groin. The patient denied any sick contacts, recent travel, camping, or any family history of autoimmune disease. Recent use of super absorbent tampons was reported.

Vital signs showed tachycardia at $120 \mathrm{bpm}$, tachypnea at 36 breaths per minute and a blood pressure supine of $131 / 68$ with a pulse of 122 , which changed with standing to $94 / 35$ and a pulse

Submitted 3 March 2005; revised 15 November 2005; accepted 21 November 2005.

From Eglin Air Force Base, Crestview, FL.

Conflict of interest: none declared.

Corresponding author: Justin M. Bailey, MD, Eglin Air Force Base, 407 Tobago Court, Crestview, FL (E-mail: justin.bailey@eglin.af.mil). of 142 . White blood cell count was 3500 cells $/ \mathrm{mm}^{3}$ (3200 to 1100 cells $/ \mathrm{mm}^{3}$ ) hemoglobin was 12 $(12-16 \mathrm{~g} / \mathrm{dL})$ and hematocrit was $36 \%$ packed red blood cells (PRBC) (36\% to $46 \%)$. Manual differential was normal.

Initial blood, urine, and cerebrospinal fluid (CSF) cultures as well as basic chemistries, and liver function tests were all within normal limits. All blood cultures were drawn for 2 separate vein puncture sites using sterile technique. Anaerobic cultures were grown in a brain, heart infusion (BHI) agar and aerobic cultures in a tripeptic soy broth. All blood cultures were kept for 5 days (92\% detection rate/culture). ${ }^{2}$ CSF was cultured out on thioglycolate, blood, chocolate, and McConkey agar and all plates were kept for 72 hours. In addition influenza, Neisseria meningitidis, streptococcus group $\mathrm{B}$, streptococcus pneumonia antigens were sent from CSF. Urine was cultured on blood and McConkey agar and kept for 48 hours.

The patient's history, signs, and symptoms were most suggestive of toxic shock syndrome. Nafcillin, doxycycline, and clindamycin were started for broad antibiotic coverage until a source was found.

Following her admission, lower leg edema worsened, and painful arthralgias developed in her hands and knees. Oliguria, hypotension, and an increasing tachycardia developed despite by mouth fluids and IV fluids. Subsequent aggressive fluid hydration was required to resolve her oliguria and hypotension. The high fever persisted for 3 days despite antipyretics and antibiotics. Repeat blood cultures were drawn every 24 hours to a total of 4 blood cultures. All cultures sent were negative. Follow up CBC counts showed an anemia with hemoglobin and hematocrit of $9 \mathrm{~g} / \mathrm{dL}$ and 27\% PRBC, respectively. Recommendation from a dermatology consult was to obtain bacterial and viral antigen titers for Rickettsia typhi, Rickettsia rickettsi, Leptospira, Ebrlichia, herpes simplex virus, influenza, and parvovirus B19. IgM and IgG for parvovirus B19 


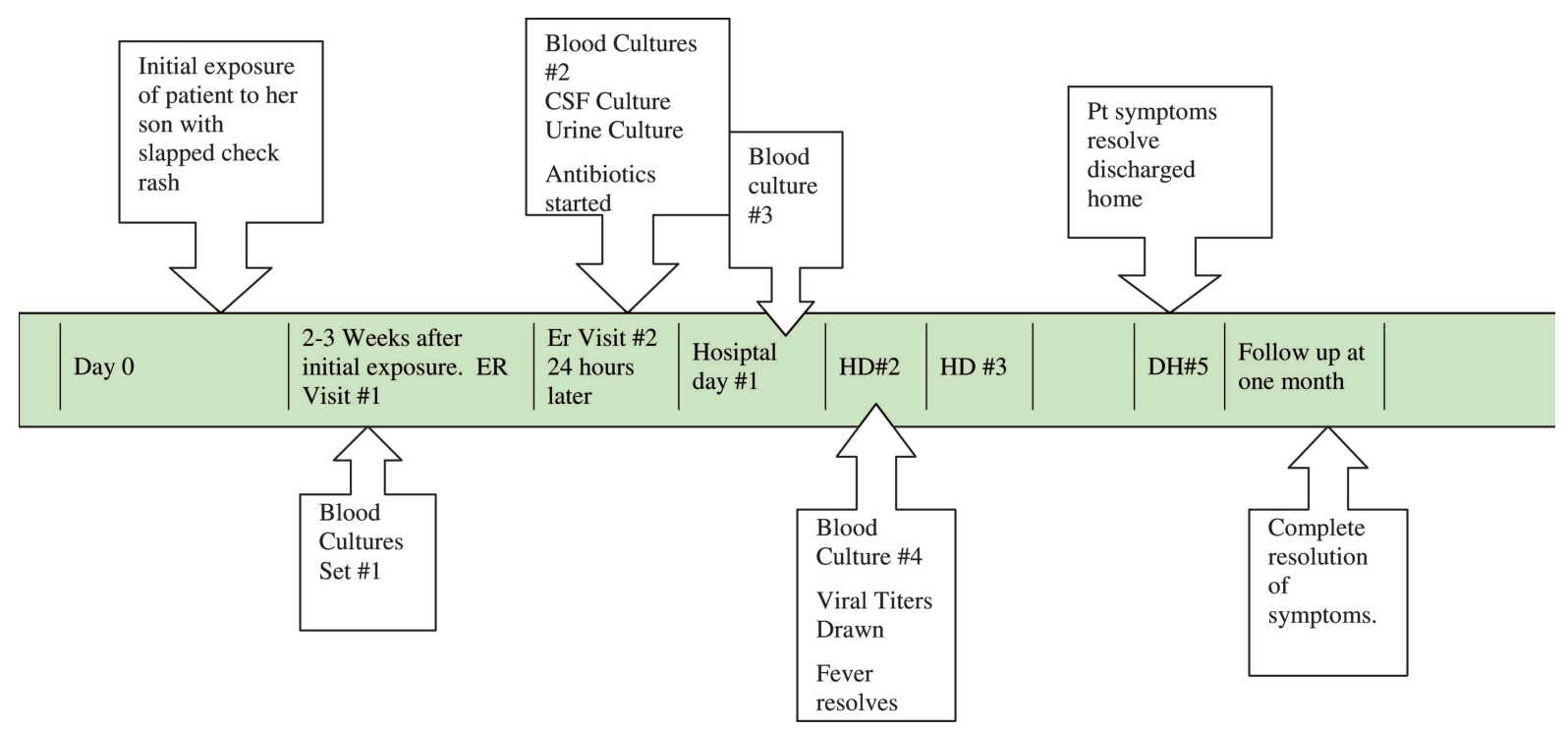

Figure 1. Disease Course.

came back significantly elevated. On further questioning the patient remembered her son had a slapped cheek rash 2 weeks earlier. Over the course of 5 days, the patient's temperature returned to normal and she was discharged. The anemia resolved 1 month after discharge and no other sequelae were noted (see Figure 1 for timeline of illness).

\section{Discussion}

Sepsis is a continuum of diagnosis progressing from systemic inflammatory response syndrome (SIRS) to septic shock. SIRS is defined as 2 of the following criteria: 1) temperature $>38^{\circ} \mathrm{C}$ or $<36^{\circ} \mathrm{C}$; 2) heart rate $>90$ beats/min; 3) respiratory rate $>20$ breaths/min or $\mathrm{PACO}_{2}<32 \mathrm{~mm} \mathrm{Hg}$; and 4) white blood cell count $>12,000$ cells $/ \mathrm{mm}^{3}$ or $<4,000$ cells $/ \mathrm{mm}^{3}$. Sepsis is SIRS plus the definitive presence of infection. Severe sepsis has the additional findings of hypotension, hypoperfusion, or organ dysfunction (such as oliguria). Septic shock is sepsis with hypotension despite adequate fluid resuscitation combined with perfusion abnormalities such as lactic acidosis, oliguria, or mental status change. ${ }^{3}$ The patient presented with all 4 of the SIRS criteria, had an infectious etiology, as well as hypotension, hypoperfusion, and organ dysfunction demonstrated by oliguria. Because she eventually responded to fluids, her final diagnosis was severe sepsis.

Bacteria account for $93 \%$ of sepsis-causing infections. ${ }^{1}$ Typically, bacterial sepsis occurs from sources in the lung, urinary tract, skin, abdomen, or central nervous system. Urinalysis, chest radiograph, CBC count, and fluid cultures remain the mainstay of diagnostic tools in looking for a source. If standard tests are negative, the differential has to be expanded.

Viruses, fungi, and protozoa become the focus of evaluation in the remaining $7 \%$ of patients. Fungus and protozoa are typically seen in immunocompromised patients. Immunocompetent patients are more likely to have a viral source. Influenza, herpes simplex virus, respiratory syncytial virus, parainfluenza, enterovirus, and adenovirus have been reported as causing sepsis. ${ }^{4-6}$ Review of the literature through MEDLINE brought up a broad differential of diseases caused by, or associated with parvovirus B19; however, multiple search strings did not reveal an association between sepsis and parvovirus B19.

Inflammatory mediators are the key players in the pathogenesis of sepsis. Exaggerated immune response from complement, coagulation, and fibrinolytic systems are thought to be the root cause of sepsis. ${ }^{7}$ Cell debris from any infection, bacterial, viral, fungal, or protozoan can activate these systems and start the cascade of inflammation that can progress to sepsis. The viral particles or the cellular debris (from red blood cell destruction) could have initiated the systemic inflammatory activation resulting in sepsis in this case.

Although parvovirus B19-causing sepsis was present in this case, traditionally, it presents as a 
self-limited illness. In children, it presents with fever, headache, and sore throat for approximately a week, which then evolves into arthralgias and erythema infectiosum rash, characterized by a slap check appearance or a reticulated rash along trunk and extremities. ${ }^{8}$ Adults more typically present with fever, arthralgias, skin rash, fatigue, and edema. ${ }^{9}$ Associated complications include congestive heart failure, myocarditis, aplastic anemia, liver failure, and meningitis. ${ }^{10,11}$

Diagnosis of parvovirus B19 is based on increased antibody titers. Elevation in the IgM titers are seen 7 to 10 days after the initial infection and can remain elevated up to 1 month. ${ }^{12,13}$ Although DNA polymerase chain reaction is available and is a more sensitive test, parvovirus B19 DNA can be found months to years later depending on the source of sample; therefore a positive test does not necessarily indicate an acute infection. ${ }^{14,15}$

Retrospectively, the patient had classic signs and symptoms of adult onset parvovirus B19. She had a probable infectious etiology presenting with fever, arthralgias, an erythema infectiosum rash, edema, anemia, and elevated IgM and IgG titers. Additional symptoms included hypotension, tachycardia, and oliguria, all indicative of sepsis.

Although initial evaluation was concerning toxic shock syndrome and the patient met most of Center for Disease Control toxic shock syndrome criteria with fever, hypotension, rash, and negative blood cultures, she ultimately failed to fulfill the multisystem criteria. This patient had 2 of the 3 required systems affected. For full detail, please see Center for Disease Control and Prevention criteria. ${ }^{16}$ All chemistry panels, liver test, and cultures were normal. CBC counts never showed leukocytosis or a left shift. The patient's presentation and laboratory tests all are suggestive of parvovirus B19 as the cause of her severe sepsis.

\section{Conclusion}

Sepsis is a condition familiar to family medicine physicians. The case presented provides the first evidence that parvovirus B19 may be an unrecognized cause of septic shock and should be added to the evaluation for viral etiologies.

\section{References}

1. Sands KE, Bates DW, Lanken PN, et al. Epidemiology of sepsis syndrome in 8 academic medical centers. Academic Medical Center Consortium Sepsis Project Working Group. JAMA 1997;278:23440.

2. Mermel LA, Maki D. Detection of bacteremia in adults: consequences of culturing an inadequate volume of blood. Ann Intern Med 1993;119:270.

3. ACCP: American College of Chest Physicians/Society of Critical Care Medicine Consensus Conference: definitions for sepsis and organ failure and guidelines for the use of innovative therapies in sepsis. Crit Care Med 1992; DA-19920706(6):864-74.

4. Pamuk ON, Pamuk GE, Celik AF, Ozturk R, Aktuglu Y. Herpes simplex virus esophagitis in an immunocompetent host with sepsis. Am J Gastroenterol 2001;96:2264-6

5. Muhe L, Tilahun M, Lulseged S, et al. Etiology of pneumonia, sepsis and meningitis in infants younger than three months of age in Ethiopia. Pediat Infect Dis J 1999;18(10 Suppl):S56-61.

6. Gatchalian SR, Quiambao BP, Morelos AM, et al. Bacterial and viral etiology of serious infections in very young Filipino infants. Pediat Inf Dis J. 1999; 18(10 Suppl):S50-5.

7. Wenzel RP, Pinsky MR, Ulevitch RJ, Young L. Current understanding of sepsis. Clin Infect Dis 1996;22:407-12.

8. Anderson LJ. Role of parvovirus B19 in human disease. Pediatr Infect Dis J 1987;6:711-8.

9. Hayakawa H, Tara M, Niina K, Osame M. A clinical study of adult human parvovirus B19 infection. Intern Med 2002;41:295-9.

10. Torok, TJ. Unusual clinical manifestations reported in patients with parvovirus B19 infection. In: Anderson LJ, Young NS, editors. Monographs in virology: human parvovirus B19. New York: Karger; 1997. p. 61.

11. Mortimer PP, Humphries RK, Moore JG, et al. A human parvovirus-like virus inhibits haematopoietic colony formation in vitro. Nature 1983;302:426-9.

12. Erdman DD. Human parvovirus B19: laboratory diagnosis. In: Anderson LJ, Young NS, editors. Monographs in virology: human parvovirus B19. New York: Karger; 1997. p. 93.

13. Erdman DD, Usher J, Tsou C, et al. Human parvovirus $\mathrm{B} 19$ specific $\operatorname{IgG}, \operatorname{IgA}$, and $\operatorname{IgM}$ antibodies and DNA in serum specimens from persons with erythema infectiosum. J Med Virol 1991;35:110-5.

14. Cassinotti P, Burtonboy G, Fopp M, Siegl G. Evidence for persistence of human parvovirus B19 DNA in bone marrow. J Med Virol 1997;53:229-32.

15. Soderlund M, von Essen R, Haapasarri J, et al. Persistence of parvovirus B19 DNA in synovial membranes of young patients with and without chronic arthropathy. Lancet 1997;349:1063-5.

16. Case definitions for infectious conditions under public health surveillance. Morb Mortal Wkly Rep 1997; 46:39. 